\title{
Principales hipótesis inmunológicas de la demodicosis canina
}

\section{Main immunological hypotheses of the canine demodicosis}

Fecha de recepción: 19 de marzo de 2018

Fecha de aprobación: 21 de mayo de 2018
Carlos Josué Cen-Cen ${ }^{1}$

Manuel Emilio Bolio-González ${ }^{2}$

Roger Iván Rodríguez-Vivas ${ }^{3}$

DOI: http://doi.org/10.19053/01228420.v15.n2.2018.8396

\section{Resumen}

La demodicosis canina es una enfermedad dermatológica común en perros; se presenta por un aumento en la proliferación de los ácaros del género Demodex spp., pero, a pesar de su alta prevalencia, su aparición y desarrollo son aún desconocidos, sin embargo, se asocian, principalmente, a una inmunodisfunción. El sistema inmune juega un papel importante tanto en el control del ácaro en animales sanos como en el desarrollo de la enfermedad en enfermos; en estos últimos se desconocen los mecanismos inmunitarios que llevan al desbalance en el sistema innato y adaptativo y a la evasión del sistema inmune por el ácaro; debido a esto se han postulado hipótesis que, en conjunto, tratan de explicar los posibles mecanismos inmunológicos. El presente artículo revisa las principales hipótesis inmunológicas relacionadas con la presentación de demodicosis en perros.

Palabras clave: demodicosis; Demodex spp.; sistema inmune.

\begin{abstract}
Canine demodicosis is a common cutaneous condition in dogs, characterized by a proliferative increase of mites from the genus Demodex. Despite its high prevalence, the onset and development of demodicosis are still unknown; however, they are primarily associated to an immune dysfunction. The immune system plays an important role controlling mites in healthy animals as well as on the development of the disease in sick animals. The immune mechanisms that lead to an unbalance in the innate and adaptive systems, as well as the mite mechanisms to evade the immune system remain unknown. Consequently,

1 Universidad Autónoma de Yucatán (Yucatán, México).

2 Ph. D. Universidad Autónoma de Yucatán (Yucatán, México). bgonza@correo.uady.mx. ORCID: 0000-0001-8702-9399.

3 Ph. D. Universidad Autónoma de Yucatán (Yucatán, México). rvivas@correo.uady.mx. ORCID: 0000-0002-3340-8059.
\end{abstract}

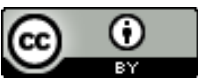


various hypotheses have been proposed to try explaining possible immune mechanisms. This paper aims to conduct an updated review of the main hypotheses related to the immune mechanisms associated to demodicosis in dogs.

Keywords: demodicosis; Demodex ssp.; immune system.

\section{Para citar este artículo:}

Cen-Cen CJ., Bolio-González ME., Rodríguez-Vivas RI. Principales hipótesis inmunológicas de la demodicosis canina. Ciencia y Agricultura. 2018; 15(2): 61-69. 


\section{Introducción}

La demodicosis canina es una enfermedad dermatológica severa que registra alta prevalencia; ocurre debido a la proliferación excesiva de ácaros del género Demodex en los folículos pilosos y glándulas sebáceas. Las principales especies de Demodex que afectan a los perros son tres: Demodex canis, $D$. injai y $D$. cornei, las cuales pueden producir signos clínicos característicos de la enfermedad, como alopecia, eritema, seborrea, hiperqueratosis folicular, costras, pústulas y dermatitis. El manejo de esta enfermedad es considerado un desafío para la Dermatología Veterinaria (1-4).

Los ácaros del género Demodex se encuentran de manera natural en la piel de sus hospederos (mamíferos), formando parte de la microbiota; en la mayoría de los casos no generan una reacción adversa en sus hospederos, pues el sistema inmunológico de estos detecta la presencia de los ácaros y ejerce un control sobre ellos sin necesidad de una respuesta inflamatoria, manteniendo un número bajo de Demodex; sin embargo, por causas aún desconocidas con exactitud, los ácaros del género Demodex proliferan, generando la enfermedad (58).

La demodicosis se asocia a perros que presentan alguna inmunodeficiencia, relacionada, generalmente, a terapias inmunosupresoras (hormonal y citotóxicas), así como a enfermedades crónico-degenerativas como la diabetes mellitus y cáncer; en cachorros, el estrés puede jugar un papel importante en la aparición de esta patología $(4,6$, 8-11). Debido a esto, algunos autores sugieren que el sistema inmune del animal juega un papel importante en la presentación de esta enfermedad y consideran que su respuesta contra el ácaro Demodex es compleja. Durante décadas se pensó que la respuesta inmune adaptativa contra la demodicosis solamente involucraba la respuesta inmune celular, debido a que la evidencia sugería que el principal mecanismo de control de ácaros Demodex era mediado por células; sin embargo, en estudios recientes se ha encontrado que la respuesta inmune humoral contra $D$. canis, mediada por Inmunoglobulinas G (IgG), juega un rol importante en el control del ácaro, por lo tanto, la respuesta inmune en la demodicosis canina involucra tanto la respuesta innata como la adaptativa humoral y celular del animal (4, 8-10).

A pesar de los avances obtenidos en el estudio de la respuesta inmune frente a Demodex, aún existen dudas acerca del rol principal del sistema inmune en torno a la aparición de la demodicosis, por lo que se han postulado algunas hipótesis que pretenden dar una explicación a la presentación de la enfermedad en mamíferos $(7,8,11)$. El objetivo del presente artículo es realizar una revisión actualizada de los principales mecanismos inmunológicos responsables de la presentación de demodicosis en perros.

\section{Demodicosis}

La demodicosis canina es, probablemente, el mejor ejemplo de enfermedad inducida por la proliferación excesiva de ácaros, en sus fases inmaduras (huevo, larva y ninfa) y adultas, la cual induce en el animal una inflamación cutánea. Sin embargo, el ácaro Demodex es considerado un habitante normal de la piel en perros y, generalmente, no induce la enfermedad en estos, pero en ciertas circunstancias se vuelve un patógeno oportunista $(4,7$, $10,12)$. El desarrollo de la enfermedad en perros se ve influenciado por numerosos factores, como defectos genéticos, alteraciones en la estructura y bioquímica de la piel, desórdenes inmunológicos, estatus hormonal, raza, edad, longitud del pelo, ciclo estral, parto, endoparásitos y enfermedades crónico-degenerativas $(9,11,12)$.

Diversos autores sugieren que un defecto inmunológico genéticamente preprogramado es el responsable de la proliferación excesiva de los ácaros en la demodicosis; no obstante, esta hipótesis no concuerda con la experiencia clínica y con los diversos reportes de publicaciones científicas, los cuales señalan que en la mayoría de los perros tratados apropiadamente no existió una recaída a la enfermedad. Así, la patogénesis exacta de la demodicosis generalizada canina es aún desconocida; algunos autores asocian los signos clínicos a una anomalía o aberración inmunológica $(3,7,11,13$, 14). 


\section{Control inmunológico del ácaro Demo- dex spp.}

En animales clínicamente sanos la población de ácaros es controlada por el sistema inmunológico; esto se da cuando el queratinocito reconoce la quitina de los ácaros por medio de sus receptores tipo Toll (TLR, su sigla en inglés), específicamente TLR2, provocando una respuesta inmune innata. Sin embargo, la respuesta innata no es la única responsable en el control del ácaro; algunas publicaciones reportan que el sistema inmune del hospedero detecta lipasas y proteasas secretadas por los ácaros, estimulando, posiblemente, la respuesta inmune adaptativa, que es más específica y efectiva para el control del ácaro Demodex, pero en perros clínicamente sanos no se han documentado anticuerpos contra Demodex, a diferencia de humanos clínicamente sanos, cuya respuesta inmune humoral juega un papel importante. En los últimos años se ha tratado de dilucidar el papel de la respuesta inmune en la demodicosis, sin tener resultados convincentes; para ello se requieren estudios que expliquen los mecanismos de control y respuesta inmune de las poblaciones de ácaros en perros sanos (Figura 1) $(7,9,13)$.

Debido a los avances en el estudio de la demodicosis canina, se han postulado diversas hipótesis, que abarcan los principales hallazgos encontrados en los estudios recientes y plantean un panorama complejo sobre la inmunología de la demodicosis.

\section{Hipótesis asociadas a la demodicosis ca- nina}

\section{A. Hipótesis inmunosupresora}

La hipótesis de que la demodicosis ocurre por supresión de la respuesta inmune se basa en que los animales y los humanos adquieren esta enfermedad cuando presentan dicha supresión o debido a una inmunodeficiencia $(6,7,11,14)$.

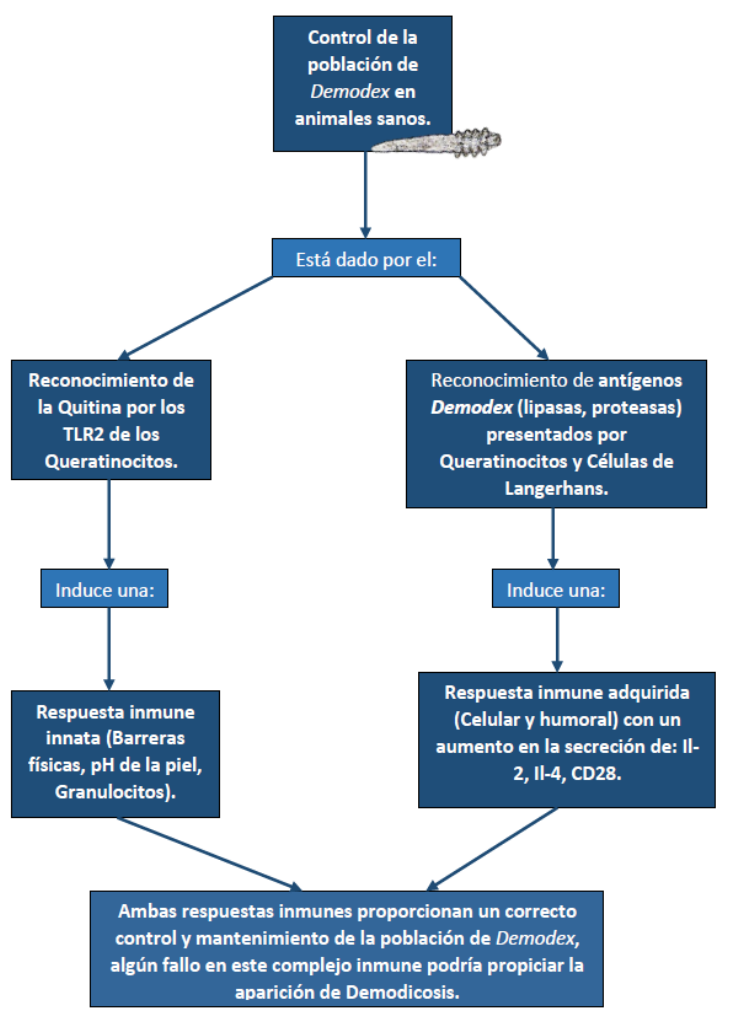

Fig. 1. Control de la población de ácaros Demodex. Modificado de: Ferrer et al. (7).

Owen, citado por Ferrer et al. (7), demostró que la inducción de demodicosis se produce por supresión de la respuesta inmune; este autor aplicó suero antilinfocitario a ocho cachorros y a cinco más los mantuvo como grupo control; al final de su experimento describió el desarrollo de demodicosis en su variante generalizada en los cachorros tratados con suero antilinfocitario, y los perros no tratados no desarrollaron la enfermedad. La proliferación excesiva de ácaros induce la secreción de factores humorales que provocan supresión de la respuesta inmune contra el ácaro, lo cual permite la proliferación de estos; sin embargo, en algunos reportes muchos perros con inmunosupresión nunca desarrollaron demodicosis, lo cual permite concluir que la inmunosupresión no es el único factor desencadenante de demodicosis y que en la aparición de la enfermedad pueden existir más de un factor asociado $(7,11)$. 
La inmunosupresión como factor desencadenante de demodicosis ha sido estudiado en ratones modificados genéticamente para hacerlos carentes de linfocitos T y células NK (Natural Killer) maduras. En este estudio se pudo concluir que el control inmune de las poblaciones de Demodex es muy complejo e incluye al sistema humoral y una coestimulación de moléculas, como la CD28, la cual tiene una participación importante en la activación de los linfocitos $T$ durante la respuesta inmune celular $(7,15)$.

Adicionalmente, en humanos y animales con alguna inmunodeficiencia asociada a una enfermedad primaria se han reportado casos de demodicosis generalizada. En el caso de perros se ha documentado la aparición de demodicosis en individuos con enfermedades crónico-degenerativas, como leishmaniosis, hipotiroidismo, hiperadrenocorticismo y neoplasias. Sin embargo, en estos estudios el mecanismo fisiopatológico para la presentación de demodicosis no ha sido estudiado $(7,9)$.

\section{B. Hipótesis del desequilibrio en las células $T$}

Estudios recientes han abordado la función de los linfocitos $\mathrm{T}$ y su impacto en el desarrollo y control de diversas enfermedades, entre las que destaca la demodicosis, en la cual los linfocitos T, específicamente CD4+, participan activamente en la disminución de las poblaciones de Demodex; sin embargo, durante la demodicosis existe un aumento en la tasa de apoptosis y en el agotamiento de las células T CD4+. El agotamiento de células $T$ es una inmunodisfunción encontrada en estadios crónicos de demodicosis generalizada, caracterizada por la pérdida progresiva de las funciones antígeno-específicas de los linfocitos T. El agotamiento de los linfocitos $T$ también ha sido descrito en infecciones virales, en enfermedades parasitarias y en neoplasias malignas. Este agotamiento presenta una disminución en la producción de citoquinas estimuladoras, como las interleucinas 2 y 21 (IL-2 e IL-21) y un aumento en la secreción de citoquinas supresoras, como es la IL-10. Por último, este desbalance de las células T proporciona una explicación razonable a la ausencia de recaídas en algunos perros después del tratamiento con lactonas macrocíclicas en demo- dicosis generalizada; esto debido a que la función principal de los acaricidas es la disminución de la carga parasitaria, lo cual permite al sistema inmune del hospedero recuperarse y volver a retomar el control en la proliferación de los ácaros $(3,7,11$, $14,16,17)$.

\section{Hipótesis de inmunosupresión regulada por citoquinas}

Las citoquinas ejercen una función específica durante los procesos de enfermedad e inflamación, y algunas, como se ha encontrado en estudios recientes, pueden tener también una función inmunosupresora, por ejemplo, el factor de crecimiento transformante beta (TGF- $\beta$ ); esta molécula biológica, aunque regula varios procesos biológicos, como pueden ser homeostasis tisular, angiogénesis, migración y diferenciación de células, también es considerada un poderoso inmunosupresor, principalmente en enfermedades crónicas. En animales con demodicosis generalizada crónica se ha encontrado un aumento en la circulación del TGF- $\beta$, el cual podría explicar el paso de la enfermedad de una etapa localizada a una generalizada. Asimismo, en estos animales se ha encontrado un aumento en la producción de TGF- $\alpha$, citoquina que no presenta un efecto inmunosupresor como la TGF- $\beta$, pero que puede desplegar la respuesta inflamatoria y provocar daño a nivel tisular; esta acción podría explicar, en parte, los signos clínicos encontrados en la demodicosis $(3,11,18,19)$. También, se ha observado en perros con demodicosis recurrente un aumento en los niveles séricos de IL-10; esta molécula es esencial en la inhibición de la síntesis de citoquinas de las células Th1, lo cual deriva en una supresión de células T; además, esta supresión afecta la capacidad de presentación de antígenos por las células presentadoras de antígenos. Los cambios en los niveles de ciertas citoquinas e interleucinas durante la demodicosis generalizada pueden explicar la aparición de la enfermedad y los hallazgos clínicos encontrados en esta $(3,11,18,19)$.

\section{Hipótesis de estrés oxidativo}

El estrés oxidativo (EO) resulta de una producción excesiva de radicales libres (oxígeno) que no son 
removidos por los antioxidantes, lo cual deriva en un daño celular. Los radicales libres asociados a EO pueden ser producidos por el sistema inmune, resultan útiles en procesos inflamatorios y tienen un efecto citotóxico contra parásitos y otros agentes patógenos. Debido al proceso citotóxico que presentan los radicales libres, se han relacionado con la presentación de signos clínicos en la demodicosis $(3,11,20,21)$. No obstante, el EO ayuda en el desarrollo de la reacción inflamatoria, pero también puede ocasionar un efecto negativo en las células del sistema inmune; esto se debe a la alta concentración de ácidos grasos poliinsaturados en estas células, los cuales son vulnerables a la peroxidación lipídica, produciendo grandes cantidades de metabolitos reactivos. Debido al daño celular inducido por el EO, las células inmunes presentan apoptosis (muerte celular), esto deriva en una disminución de las células inmunes y, por lo tanto, en una inmunodepresión. El incremento de apoptosis celular se ha documentado en perros con demodicosis; asimismo, el balance oxidativo/ antioxidativo puede estar involucrado en la progresión de esta enfermedad $(3,11,20,21)$.

\section{E. Hipótesis del sistema colinérgico}

Estudios recientes han postulado un efecto directo del sistema parasimpático en el control y regulación de la inmunidad, así como en la modulación de la inflamación mediante la producción de citoquinas derivadas de la vía colinérgica. Esta vía presenta como molécula principal la acetilcolina (ACh), que tiene como función principal la estimulación neuronal del sistema nervioso y también participa como mediadora de la inflamación. La ACh actúa inhibiendo la producción del Factor de Necrosis Tumoral alfa (TNF- $\alpha$ ), de la Interleucina-1 $\beta$ (IL-1 $\beta$ ) y del factor inhibidor de la migración de macrófagos, los cuales son catalogados como citoquinas proinflamatorias; asimismo, no promueve el aumento de citoquinas inmunosupresoras, como la interleucina 10 (IL-10). En términos sencillos, la ACh juega un papel importante no solo como neurotransmisor, sino también como mediador en el proceso inflamatorio $(3,11,14)$. La ACh no puede ser medible a nivel sérico, pero existe una enzima, denominada acetilcolinesterasa, que regula la acción del neurotransmisor durante las sinapsis neuronales y que es considerada un biomarcador indirecto de la ACh. Debido a esto, Kumari et al. (3) analizaron muestras de suero de varios perros y midieron la actividad de la acetilcolinesterasa, y encontraron que durante la demodicosis existía un aumento en la actividad de esta enzima, lo que podría sugerir una sobreproducción de ACh, lo cual se traduce en una inmunodepresión por la falta síntesis de citoquinas proinflamatorias. Asimismo, estos autores observaron una variación en la actividad de la acetilcolinesterasa asociada al tipo de demodicosis (localizada o generalizada); este hallazgo propone que la activación de la vía colinérgica en los perros se asocia a una respuesta fisiológica aún no comprendida, y esto puede propiciar el progreso de la demodicosis, pasando de una demodicosis localizada a una generalizada con o sin problemas bacterianos secundarios (Figura 2) (3). Esto pone de manifiesto que la ACh puede jugar un rol principal en el desarrollo de la demodicosis; sin embargo, esta vía no es la única que inmunodeprime al animal, y se ha postulado que una aberración en la inmunidad y el incremento en la producción de citoquinas inmunosupresoras juegan un papel fundamental $(3,7,11,14)$. 


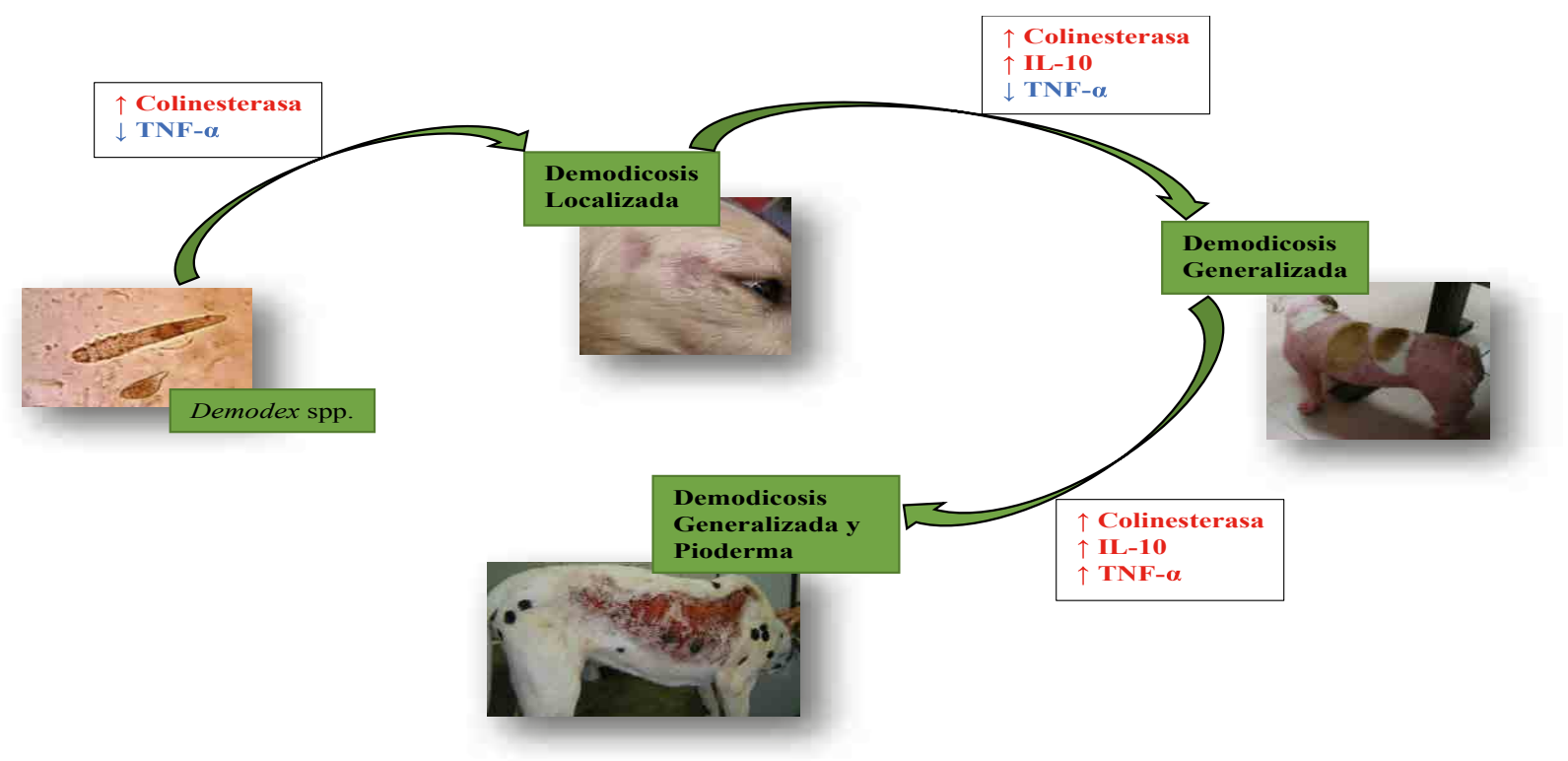

Fig. 2. Desarrollo de demodicosis localizada y generalizada en perros por efecto de la actividad de la acetilcolina. Modificado de: Kumari et al. (3).

\section{F. Hipótesis del efecto en los receptores tipo Toll}

Los receptores tipo Toll (TLRs) son proteínas transmembranales encontradas en la membrana de las células y en algunos organelos; su función principal es activar la inflamación e inducir una respuesta adaptativa por medio del reconocimiento de ciertas estructuras bacterianas, virales y parasitarias (18). En los últimos años estos receptores han ganado una atención especial debido a su importancia en la lucha contra enfermedades parasitarias, y en dermatología veterinaria estas proteínas son estudiadas buscando una relación especial con el inicio, desarrollo y mantenimiento de la demodicosis en perros. Esto complementa los conocimientos que se tienen de la inmunología de la enfermedad, la cual dictamina que los ácaros Demodex spp. son reconocidos por los TLRs de los queratinocitos y estos inducen una inmunidad innata, con el fin de detener la sobreproducción de los ácaros en la piel de los perros $(7,9,18)$. Sin embargo, este conocimiento de la función de los receptores solamente se había asociado al control de los ácaros en animales sanos, pero en animales con demodicosis se encontraron cambios importantes que reafirman el papel que juegan los TLRs en el desarrollo de la enfermedad. Estos cambios son mencionados en el estudio realizado por Kumari et al. (18), en donde observaron que en animales con demodicosis existía una elevación en la expresión de los TLRs tipo 2 en las células mononucleares (linfocitos y monocitos) y, también, una disminución en la expresión de TLRs tipo 3 y 4 ; este efecto en la expresión de los TLRs puede deberse a la acción de los ácaros. Kumari et al. (18) explican que el aumento de los TLRs tipo 2 se puede asociar al inicio de la enfermedad y a la aparición de signos clínicos; pero, a pesar de esto, al existir igualmente una disminución en la expresión de los receptores tipo 3 y 4 , el proceso inflamatorio se ve disminuido o mediado, debido a que estos estimulan la producción de Il-4, 5 y 13 (Figura 3). La pobre expresión de estos receptores se ha asociado a la estrategia que pueden llevar a cabo los ácaros Demodex spp. para evadir la respuesta inmune del hospedador. Por último, aún no se sabe cómo el ácaro estimula o disminuye la producción de los receptores tipo Toll en el proceso de la enfermedad $(7,9,18)$. 


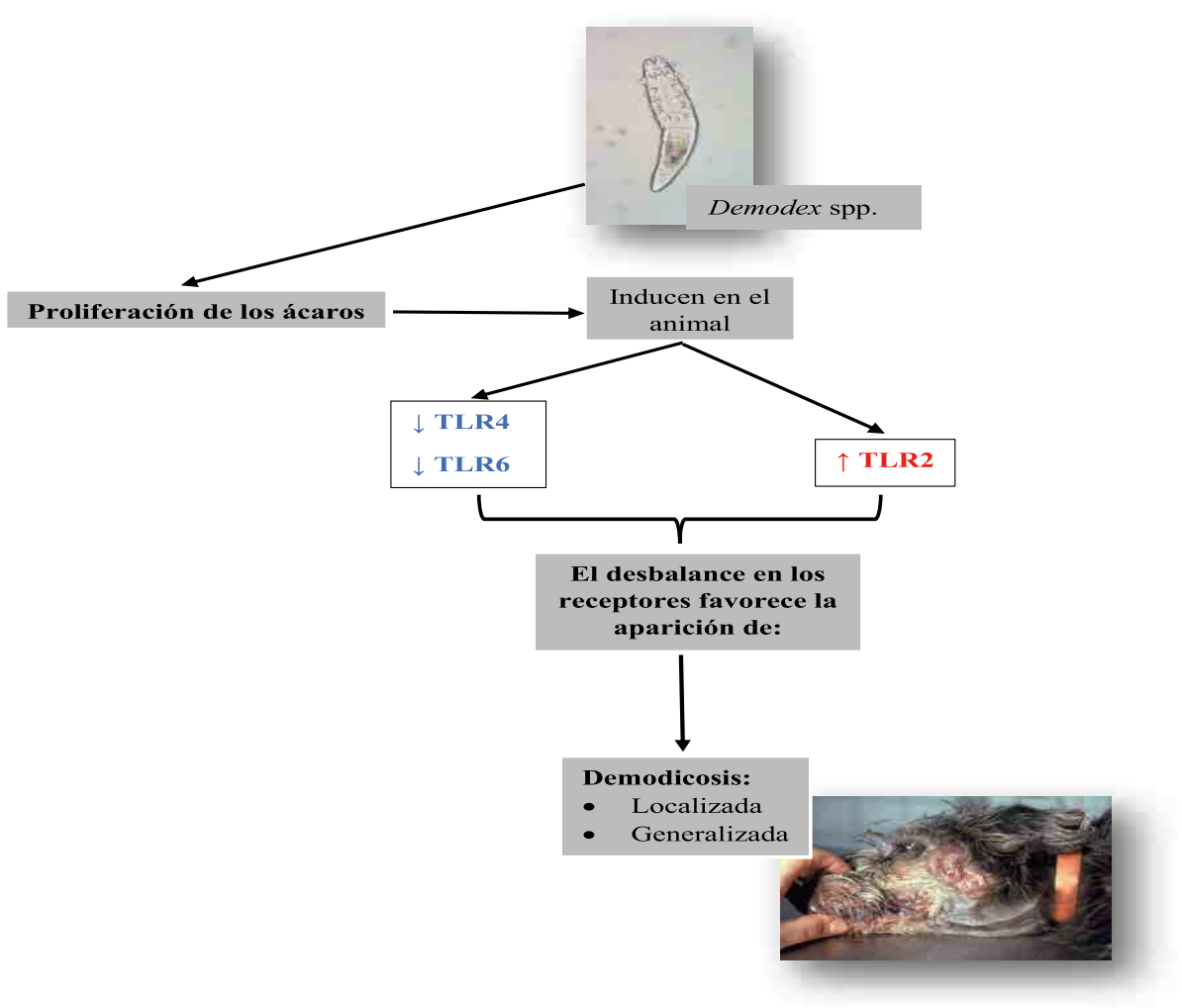

Fig. 3. Regulación de los TLRs por los ácaros Demodex spp. para inducir demodicosis canina. Modificado de: Kumari et al. (18).

\section{Conclusiones}

La demodicosis es una enfermedad de gran importancia para la medicina veterinaria; a pesar de ser una enfermedad con alta prevalencia, su aparición y desarrollo en los perros aún son desconocidos. Debido a esto, se han postulado diversas hipótesis que tratan de dar una explicación científica, fisiopatológica e inmunológica a todo el proceso de la enfermedad; algunas de estas hipótesis mencionan los mecanismos básicos por los cuales el sistema inmune controla al ácaro, y otras profundizan en las principales condiciones involucradas en la evasión del sistema inmune por los ácaros y en la proliferación de estos. Sin embargo, las diversas hipótesis inmunológicas planteadas por sí solas no logran dilucidar la causa exacta de la proliferación de los ácaros, pero en conjunto logran dar una respuesta más sólida a esta preguntan y sientan las bases para otros estudios enfocados en el diagnóstico, pero, sobre todo, en el tratamiento de la enfermedad.

\section{Referencias}

(1) Ordeix L, Bardagí M, Scarampella F, Ferrer L, Fondati A. Demodex injai infestation and dorsal greasy skin and hair in eight wirehaired for terrier dogs. Vet. Dermatol. 2009; 20: 267-272. DOI: https://doi.org/10.1111/j.1365-3164.2009.00755.x.

(2) Molecular study on Three morphotypes of Demodex mites (Acarina: Domodicidae) from dogs.

(3) Kumari P, Nigam R, Singh A, Nakade UP, Sharma A, Garg SK, Singh SK. Demodex canis regulates cholinergic system mediated immunosuppressive pathways in canine demodicosis. Parasitol. 2017; 144 (10): 1412-1416. DOI: https://doi.org/10.1017/ S0031182017000774.

(4) Moskvina TV. Two morphologically distinct forms of Demodex mites found in dogs with canine demodicosis from vladivostok, Russia. Acta Vet. 2017; 67(1): 82-91. DOI: https://doi. org/10.1515/acve-2017-0008.

(5) Chen Y, Lin R, Zhou D, Song H, Chen F, Yuan Z, Zhu X, Weng Y, Zhao G. Prevalence of Demodex infection in pet dogs in Southern China. Afr. J. Microbiol. Res. 2012; 6(6): 1279-1282. DOl: https://doi.org/10.5897/AJMR11.1530. 
(6) Mueller RS, Bensignor E, Ferrer L, Holm B, Lemarie S, Paradis M, Shipstone MA. Treatment of demodicosis in dogs: 2011 clinical practice guidelines. Vet. Dermatol. 2012; 23(2): 86-96. DOI: https://doi.org/10.1111/j.1365-3164.2011.01026.x.

(7) Ferrer L, Ravera I, Silbermayr K. Immunology and pathogenesis of canine demodicosis. Vet. Dermatol. 2014; 25(5): 427-435. DOI: https://doi.org/10.1111/vde.12136.

(8) Ravera I, Ferreira D, Gallego LS, Bardagí M, Ferrer L. Serum detection of IgG antibodies against Demodex canis by western blot in healthy dogs and dogs with juvenile generalized demodicosis. Res. Vet. Sci. 2015; 101: 161-164. DOI: https://doi. org/10.1016/j.rvsc.2015.06.011.

(9) Ravera I. Deconstructing canine demodicosis. Tesis de Doctorado.Facultad de Medicina Veterinaria, Departamento de Medicina y Cirugía Animal, Universidad Autónoma de Barcelona, 2015.

(10) Sgarbossa RS, Sechi GV, Pacheco BD, Lucina SB, Paulo MR, Monti FD, Farias MR. The epidemiological and clinical aspects of Demodex injai demodicosis in dogs: a report of eight cases. Semin. Cienc. Agrar. 2017; 38(5): 3387-3393. DOI: https://doi. org/10.5433/1679-0359.2017v38n5p3387.

(11) Singh SK, Dimri U. The immuno-pathological conversions of canine demodicosis. Vet. Parasitol. 2014; 203: 1-5. DOI: https:// doi.org/10.1016/j.vetpar.2014.03.00.

(12) Scott DW, Miller WH, Griffin CE, Scott DW, Miller WH, Griffin CE. Muller \& Kirk's Small Animal Dermatology. Chapter 6. Seven Edition. Missouri, USA 2013; pp. 432-500.

(13) Kumari P, Nigam R, Choudhury S, Singh SK, Yadav B, Kumar D, Garg SK. Demodex canis targets TLRs to evade host immunity and induce canine demodicosis. Parasite. Immunol. 2018; 40(3): 1-5. DOI: https://doi.org/10.1111/pim.12509.

(14) Singh SK, Dimri U, Sharma MC, Swarup D, Sharma B, Pandey $\mathrm{HO}$, Kumari P. The role of apoptosis in immunosuppression of dogs with demodicosis. Vet. Immunol. Immunopathol. 2011; 144: 487-492. DOI: 10.1016/j.vetimm.2011.08.008.

(15) Saavedra-Hernández D. La molécula CD28 y su función en la activación de células T. Rev. Cuba Hematol. Inmunol. Hemoter. 2013; 29(4): 359-367.

(16) Oliveira CD, Larsson CE, Camargo MM. Longitudinal assessment of T-lymphocyte subpopulations during generalized demodicosis in dogs and their relationship with remission. Vet. Dermatol. 2015; 26: 18-22. DOI: https://doi.org/10.1111/vde.12183.

(17) Huisinga M, Failing K, Reinacher M. MHC class II expression by follicular keratinocytes in canine demodicosis-An immunohistochemical study. Vet. Immunol. Immunopathol. 2007; 118: 210-220. DOI: https://doi.org/10.1016/j.vetimm.2007.05.020.

(18) Yarim GF, Yagci BB, Yarim M, Sozmen M, Pekmezci D, Cenesiz S, Pekmezci GZ, Karaca E. Serum concentration and skin tissue expression of insulin-like growth factor 2 in canine generalized demodicosis. Vet. Dermatol. 2015; 26: 421-425. DOI: https:// doi.org/10.1111/vde.12270.

(19) Yarim GF, Yagci BB, Ciftci G. Increased circulating concentracions of PDGF-BB and TGF- -1 in canine generalised demodicosis. Revue. Méd. Vét. 2013; 164(1): 13-17.
(20) Martínez-Subiela S, Bernal LJ, Tvarijonaviciute A, Garcia-Martinez JD, Tecles F, Cerón JJ. Canine demodicosi: the relationship between response to treatment of generalised disease and markers for inflammation and oxidative status. Vet. Dermatol. 2014; 25: 72-76. DOI: https://doi.org/10.1111/vde.12108.

(21) Dimri U, Ranjan R, Kumar N, Sharma MC, Swarup D, Sharma B, Kataria M. Changes in oxidative stress indices, zinc and copper concentrations in blood in canine demodicosis. Vet. Parasitol. 2008; 154: 98-102. DOI: https://doi.org/10.1016/j.vetpar.2008.03.001. 
